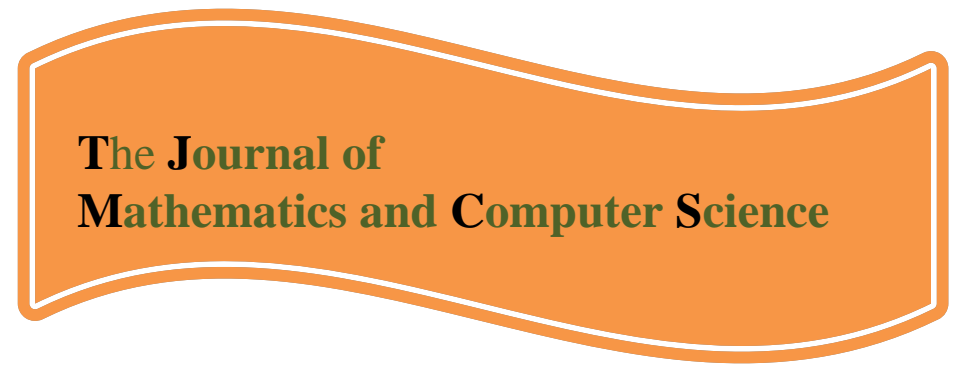

Available online at

\title{
http://www.TJMCS.com
}

The Journal of Mathematics and Computer Science Vol .2 No.3 (2011) 417-424

\section{Fuzzy path planning in a plane with stationary obstacles}

\author{
M. Zamirian ${ }^{1 *}$, A. V. Kamyad ${ }^{2}$ \\ ${ }^{1}$ Islamic Azad University, Bojnourd Branch, Department of Mathematics, Bojnourd, Iran, \\ ${ }^{2}$ Department of Mathematics, Ferdowsi University of Mashhad, Mashhad, Iran
}

Received: August 2010, Revised: November 2010

Online Publication: January 2011

\begin{abstract}
In this paper a new approach for finding optimal path planning in a plane with stationary obstacles is discussed. At first, we consider a movable rigid object in a plane with stationary obstacles. The goal is to find the shortest path planning which brings rigid object from a given initial point to a given final point such that the length of path be minimized and distance between object and obstacles be maximized. By considering the length of path and the distance between rigid object with obstacles as objective functions, we obtain a multi-objective problem. Because of the imprecise nature of decision maker's judgment, these multiple objectives are viewed as fuzzy variables. Then we determine intervals for the value of objective functions such that these intervals for the distance between rigid object and obstacles are given by decision maker, and for the length of path is achieved by solving two optimal nonlinear problems (ONP). Now, we define a decreasing or increasing membership function for any objective functions on achieved intervals. Then the optimal
\end{abstract}

\footnotetext{
* Corresponding author.

E-mail address: zamirianm@yahoo.com (Mohammad Zamirian)
} 
policy is to find an optimal path which maximize all of membership functions, simultaneously. After a little calculation, we obtain an ONP. By solving this ONP, a (local) Pareto optimal solution for original goal is attained. Numerical example is also given.

Keywords: Optimal path planning; multi-objective problem; Optimal non-linear problem; Pareto optimal solution.

\section{1. introduction}

The problem of finding an optimal path planning for a movable rigid object in a plane with stationary obstacles is one of the most applicable problems, especially in robot and recently surgery planning and etc. Latombe [1] has gathered novel methods for path planning in the present of obstacles and some extensions of them. In reference [2] two novel approaches, constrained optimization and semi-infinite constrained optimization, for unmanned under water vehicle are considered. In reference [3] is presented a new approach based on measure theory for finding approximation optimal path planning problem in the present of obstacles. In all of above references the distance between rigid object and obstacles are assumed crisp values. But, in practice we desire to achieve the shortest path with the greatest distance between rigid object and obstacles, simultaneously. All of these objectives are contradictory. Then, we need a multi-objective decision making technique to look for a satisfying solution from these conflict objectives. Optimization for a multi-objective problem is a procedure looking for a compromise policy. The result, called a Pareto optimal that consists of an infinite number of alternatives. In references [4,5] there are many methods according to different criteria. For example, Cohon [5] categorized two methods generating and preference-base. The generating methods produce a set of Pareto optimal and then the decision maker select one of them on a basis of subjective value judgment. The preference-base methods, contain decision maker's preference as the solution process goes on, and the best solution of decision maker's preference is selected. Thus, all of these multi-objective optimization methods for finding a Pareto optimal solution are filled with fuzzy properties [6]. Then we consider the length of path planning and the distance between rigid object and obstacles as objective functions which are fuzzy goals. Now we determine intervals for the value of objective functions such that these intervals for the distance between object and obstacles are given and for the shortest path is achieved by solving two optimal non-linear problems (ONP). Now, we defined a decreasing or increasing membership function for any objective functions on achieved intervals. Then, the optimal policy is to find an optimal path planning which maximize all of membership functions, simultaneously. After a little calculation, we attain an ONP. Finally, by solving ONP we achieve the approximation of a (local) Pareto optimal solution for original problem such that if the solution of ONP is unique, a (local) Pareto optimal solution is attained.

\section{Problem statement}

Consider a movable rigid object as a disk with $r$ radius and $x(t)=\left(x_{1}(t), x_{2}(t)\right)$ center in a plane with stationary obstacles which are disks with $r_{i}$ radius and $\left(\alpha_{i}, \beta_{i}\right)$ centers, $i=1, \ldots, q$. Set 
$I(\dot{x}(t))=\int_{0}^{T} \sqrt{\dot{x}_{1}^{2}(t)+\dot{x}_{2}^{2}(t)} d t$,

Where objective function $I(\dot{x}(t))$ is the length of the path which must be minimized.

Now, the distance between object and the $i$ th obstacle is is defined as follows:

$\varphi_{i}\left(x\left(t_{i x(.)}\right)\right)=\min _{t \in[0, T]} \varphi_{i}(x(t))$,

where $t_{i x(.)}$ is the time in $[0, T]$ that minimized $\varphi_{i}(x(t))$, clearly this time depends on $x($.$) .$ Then, $\varphi_{i}\left(x\left(t_{i x(.)}\right)\right)$ should be maximized to obtain a path as safe as possible.

Set $\Omega=\left\{x(t)=\left(x_{1}(t), x_{2}(t)\right) \mid x(0)=x_{i}, x(T)=x_{f}\right\}$, where $x_{i}$ and $x_{f}$ as the first and final points are given.

Now, the goal is to find an optimal path planning which minimizes the length of path, $I\left(x\left(t_{f}\right)\right)$, and maximizes the distance between object A and obstacle $i, \varphi_{i}\left(x\left(t_{i x(.)}\right)\right), i=1, \ldots, q$.

That is

$$
\left\{\begin{array}{l}
\min _{x(.) \in \Omega} I\left(x\left(t_{f}\right)\right), \\
\max _{x(.) \in \Omega} \varphi_{i}\left(x\left(t_{i x(.)}\right)\right), i=1,2, \ldots, q .
\end{array}\right.
$$

or in a more compact form

$$
\min _{x(.) \in \Omega} Z(x(.))=\left\{I(\dot{x}(.)),-\varphi_{1}(x(.)), \ldots,-\varphi_{q}(x(.))\right\}
$$

These objective functions are conflicted with together. The optimization of one objective implies the sacrifice of other target. Therefore, we must make some compromise among these goals. In contrast to the optimality used in single objective optimization problems, Pareto optimality characterizes the solutions in a multi-objective optimization problems $[4,5]$.

Definition 1. $x^{*}(t) \in \Omega$ is said to be Pareto optimal for problem (1), if and only if there are not $x(t) \in \Omega$ such that $I(\dot{x}(t)) \leq I\left(\dot{x}^{*}(t)\right)$ and $-\varphi_{i}(x(t)) \leq-\varphi_{i}(x(t))$ for all $i \in\{1, \ldots, q\}$ and for some $j \in\{1, \ldots, q\},-\varphi_{i}(x(t))<-\varphi_{i}(x(t))$ or $I(\dot{x}(t))<I\left(\dot{x}^{*}(t)\right)$.

From the above definition, the number of solutions satisfying Pareto optimality in a multiobjective optimization problem can be infinite. It is difficult to achieve a solution of Pareto optimal solutions without sufficient knowledge of objective. Then, we define a fuzzy problem for finding a Pareto optimal solution.

\section{Formulation of the fuzzy problem}

By determining intervals $\left[d_{i}^{l}, d_{i}{ }^{u}\right], i=1, \ldots, q$, by decision maker, and solving the following of ONPs, interval $\left[I_{1}, I_{2}\right]$ for the length of the shortest path is obtained. 
$\min I_{1}(\dot{x}(t))=\int_{0}^{T} \sqrt{\dot{x}_{1}^{2}(t)+\dot{x}_{2}^{2}(t)} d t$

$$
\begin{aligned}
& \varphi_{i}(x(t)) \geq d_{i}^{l} \quad i=1, \ldots, q \\
& x(t) \in \Omega
\end{aligned}
$$

$\min I_{2}(\dot{x}(t))=\int_{0}^{T} \sqrt{\dot{x}_{1}^{2}(t)+\dot{x}_{2}^{2}(t)} d t$

$$
\begin{aligned}
& \varphi_{i}(x(t)) \geq d_{i}{ }^{u} \quad i=1, \ldots, q \\
& x(t) \in \Omega
\end{aligned}
$$

Now, we determine fuzzy goals $\tilde{I}$ and $-\tilde{\varphi}_{i}, i=1, \ldots, q$ on intervals $\left[I_{1}, I_{2}\right]$ and $\left[-d_{i}{ }^{u},-d_{i}{ }^{l}\right]$ for Objective functions $I(\dot{x}(t))$ and $-\varphi_{i}(x(t))$, respectively.

$\mu_{\tilde{I}}(I(\dot{x}()))=.\left\{\begin{array}{cc}1 & I(\dot{x}(.))<I_{1} \\ 1-\left(I(x(.))-I_{1}\right) /\left(I_{2}-I_{1}\right) & I_{1} \leq I(\dot{x}(.)) \leq I_{2} \\ 0 & I(\dot{x}(.))>I_{2}\end{array}\right.$

$\mu_{-\tilde{\varphi}_{i}}\left(-\varphi_{i}(x()).\right)=\left\{\begin{array}{lc}1 & -\varphi_{i}(x(.))<-d_{i}^{u} \\ 1-\left(d_{i}^{u}-\varphi_{i}(x(.))\right) /\left(d_{i}^{u}-d_{i}^{l}\right) & -d_{i}^{u} \leq-\varphi_{i}(x(.)) \leq-d_{i}^{l} ; i=1, \ldots, q . \\ 0 & -\varphi_{i}(x(.)) \geq-d_{i}^{l}\end{array}\right.$

Where $\mu_{\tilde{I}}$ and $\mu_{-\tilde{\varphi}_{i}}, i=1, \ldots, q$, on intervals $\left[I_{1}, I_{2}\right]$ and $\left[-d_{i}{ }^{u},-d_{i}^{l}\right]$ are strictly monotonic decreasing, respectively. The original problem (1) is now equivalent to look for a suitable path planning policy that can provide the maximal degree of membership for the below multiple fuzzy objectives.

$\max _{x(t) \in \Omega} \mu(x(t))=\left\{\mu_{\tilde{I}}(I(x(t))), \mu_{-\tilde{\varphi}_{1}}\left(-\varphi_{1}(x(t))\right), \ldots, \mu_{-\tilde{\varphi}_{q}}\left(-\varphi_{q}(x(t))\right)\right\}$

The fuzzy optimization problem (4) is equivalent to the following problem:

$$
\max _{x(t) \in \Omega} \mathrm{T}\left\{\left\{\mu_{\tilde{I}}(I(x(t))), \mu_{-\tilde{\varphi}_{1}}\left(-\varphi_{1}(x(t))\right), \ldots, \mu_{-\tilde{\varphi}_{q}}\left(-\varphi_{q}(x(t))\right)\right\}\right\} .
$$

Where $\mathrm{T}$ is a T-norm. By using Zadeh-min as a T-norm, we have:

$$
\max _{x(t) \in \Omega} \min \left\{\left\{\mu_{\tilde{I}}(I(x(t))), \mu_{-\tilde{\varphi}_{1}}\left(-\varphi_{1}(x(t))\right), \ldots, \mu_{-\tilde{\varphi}_{q}}\left(-\varphi_{q}(x(t))\right)\right\}\right\},,
$$

or 
$\max \alpha$

$$
\begin{aligned}
& \mu_{\tilde{I}}(I(x(t))) \geq \alpha \\
& \mu_{-\tilde{\varphi}_{i}}\left(-\varphi_{i}(x(t))\right) \geq \alpha \quad ; i=1, \ldots, q \\
& x(t) \in \Omega
\end{aligned}
$$

After a little calculation, we achieve the following ONP:

$\max \alpha$

$$
\begin{aligned}
& \int_{0}^{T} \sqrt{\dot{x}_{1}^{2}(t)+\dot{x}_{2}{ }^{2}(t)} d t \leq I_{2}-\left(I_{2}-I_{1}\right) \alpha \\
& \varphi_{i}(x(t)) \geq d_{i}^{l}+\left(d_{i}^{u}-d_{i}^{l}\right) \alpha ; i=1, \ldots, q \\
& x(t) \in \Omega
\end{aligned}
$$

Theorem 1 . Let $x^{*}$ (.) be a (local) optimal solution for ONP (5) such that $\mu_{\tilde{I}}$ and $\mu_{-\tilde{\varphi}_{i}}, i=1, \ldots, q$, beings decreasing membership function, then $x^{*}(t)$ is a (local) Pareto for problem (1) (original problem) if $x^{*}(t)$ be the unique solution of problem (5)

Proof. See [7].

\section{The solution of ONP's}

There are many methods for solving ONP (2),(3) and (5) [1-3]. For example Borzabadi [3] defines the artificial control function $u(t)$ as $\dot{x}(t)=u(t)$ and achieves an optimal control problem. Then, by using of measure theory that was established by Rubio [8], obtains an approximation solution. But, we use a new approach for solving these ONPs.

Theorem 2. $\varphi_{i}(x(t)) \geq d_{i}^{l}$ if and only if $\int_{0}^{T}\left|\varphi_{i}(x(t))-d_{i}^{l}-\right| \varphi_{i}(x(t))-d_{i}^{l} \| d t=0$.

Proof. The proof is trivial.

By using theorem (2), the ONP (2) is equivalent to the following ONP:

$$
\begin{aligned}
\min & I_{1}(\dot{x}(t))=\int_{0}^{T} \sqrt{\dot{x}_{1}^{2}(t)+\dot{x}_{2}^{2}(t)} d t \\
& \int_{0}^{T}\left|\varphi_{i}(x(t))-d_{i}^{l}-\right| \varphi_{i}(x(t))-d_{i}^{l}|| d t=0 \quad ; \quad i=1, \ldots, q \\
& \left(x_{1}(0), x_{2}(0)\right)=x_{i},\left(x_{1}(T), x_{2}(T)\right)=x_{f}
\end{aligned}
$$

Now, we partition interval $[0, T]$ to $n$ equal parts where $n$ is arbitrary fixed positive integer, and the first derivative is approximated as follows: 
$\dot{x}_{j}\left(t_{k}\right)=\left(x_{j}\left(t_{k+1}\right)-x_{j}\left(t_{k}\right)\right) / \Delta t_{k}=n\left(x_{j}\left(t_{k+1}\right)-x_{j}\left(t_{k}\right)\right) ; j=1,2 \quad k=0, \ldots, n-1$,

where $t_{0}=0$ and $t_{n}=T$. Thus, by substituting in the problem (6), we have:

$$
\begin{gathered}
\min I_{1}(\dot{x}(t))=\sum_{k=0}^{n-1}\left(\left(x_{1}\left(t_{k+1}\right)-x_{1}\left(t_{k}\right)\right)^{2}+\left(x_{2}\left(t_{k+1}\right)-x_{2}\left(t_{k}\right)\right)^{2}\right)^{1 / 2} \\
\sum_{k=0}^{n-1}\left|\varphi_{i}\left(x\left(t_{k}\right)\right)-d_{i}^{l}-\right| \varphi_{i}\left(x\left(t_{k}\right)\right)-d_{i}^{l}||=0 ; \quad i=1, \ldots, q \\
\left(x_{1}(0), x_{2}(0)\right)=x_{i},\left(x_{1}(T), x_{2}(T)\right)=x_{f}
\end{gathered}
$$

Remark. As we know, an approximate value of integral $\int_{a}^{b} f(x) d x$ is $(b-a) f(a)$.

With the same method, the two ONPs (3) and (5) are obtained as follows:

$$
\begin{gathered}
\min I_{2}(\dot{x}(t))=\sum_{k=0}^{n-1}\left(\left(x_{1}\left(t_{k+1}\right)-x_{1}\left(t_{k}\right)\right)^{2}+\left(x_{2}\left(t_{k+1}\right)-x_{2}\left(t_{k}\right)\right)^{2}\right)^{1 / 2} \\
\sum_{k=0}^{n-1}\left|\varphi_{i}\left(x\left(t_{k}\right)\right)-d_{i}^{u}-\right| \varphi_{i}\left(x\left(t_{k}\right)\right)-d_{i}^{u}||=0 ; i=1, \ldots, q \\
\left(x_{1}(0), x_{2}(0)\right)=x_{i},\left(x_{1}(T), x_{2}(T)\right)=x_{f}
\end{gathered}
$$

$\min \alpha$

$$
\begin{aligned}
& \sum_{k=0}^{n-1}\left(\left(x_{1}\left(t_{k+1}\right)-x_{1}\left(t_{k}\right)\right)^{2}+\left(x_{2}\left(t_{k+1}\right)-x_{2}\left(t_{k}\right)\right)^{2}\right)^{1 / 2} \leq I_{2}-\left(I_{2}-I_{1}\right) \alpha \\
& \sum_{k=0}^{n-1}\left|\varphi_{i}\left(x\left(t_{k}\right)\right)-d_{i}^{l}-\left(d_{i}^{u}-d_{i}^{l}\right) \alpha-\right| \varphi_{i}\left(x\left(t_{k}\right)\right)-d_{i}^{l}-\left(d_{i}{ }^{u}-d_{i}^{l}\right) \alpha \mid=0 ; i=1, \ldots, q \\
& \quad\left(x_{1}(0), x_{2}(0)\right)=x_{i}, \quad\left(x_{1}(T), x_{2}(T)\right)=x_{f}
\end{aligned}
$$

Theorem 3. The ONPs (7),(8) and (9) are equivalent to the ONPs (2),(3) and (5), respectively, if in the ONPs (7),(8) and (9) $n$ tend to infinity.

Proof. Is trivial.

Thus, we can solve the ONPs (7),(8) and (9) by many packages such as Lingo, Matlab, Gino and etc. Finally, by solving the ONPs, we recognize the value of unknown pairs $\left(x_{1}\left(t_{k}\right), x_{2}\left(t_{k}\right)\right), k=0, \ldots, n$ and construct optimal path planning by joining points $\left(x_{1}\left(t_{k}\right), x_{2}\left(t_{k}\right)\right), k=0, \ldots, n$ together. Also, we may fit a curve by these points as an optimal path planning.

\section{Numerical example}


Example . Consider a movable rigid object and five stationary obstacles as disks in a plane with below information:

Object : $\quad r=0.01$,

Obstacles:

$$
\begin{aligned}
& r_{i}=1 / 8, i=1, \ldots, 5,\left(\alpha_{1}, \beta_{1}\right)=(0.5,0.5),\left(\alpha_{2}, \beta_{2}\right)=(0.7,0.85), \\
& \left(\alpha_{3}, \beta_{3}\right)=(0.2,0.2),\left(\alpha_{4}, \beta_{4}\right)=(0.3,0.8),\left(\alpha_{5}, \beta_{5}\right)=(0.85,0.2) \\
& d_{i}^{l}=0, i=1, \ldots, 5, \quad d_{1}{ }^{u}=0.2, d_{2}{ }^{u}=0.1, d_{3}{ }^{u}=0.2, d_{4}{ }^{u}=0.1, \\
& d_{5}{ }^{u}=0.015, x_{i}=(0,0), x_{f}=(1,1), n=40 .
\end{aligned}
$$

By solving the ONP (7) we achieve $I_{1}=1.4597$ such that in reference [3] is 1.4641 . By solving the ONP (8) we obtain $I_{2}=1.7298$. Finally, by solving the ONP (9), the optimal path planning with the length $I^{*}=1.5748$ is achieved $\left(\alpha^{*}=0.5741\right)$. The following figure shows the optimal path planning for the ONPs.

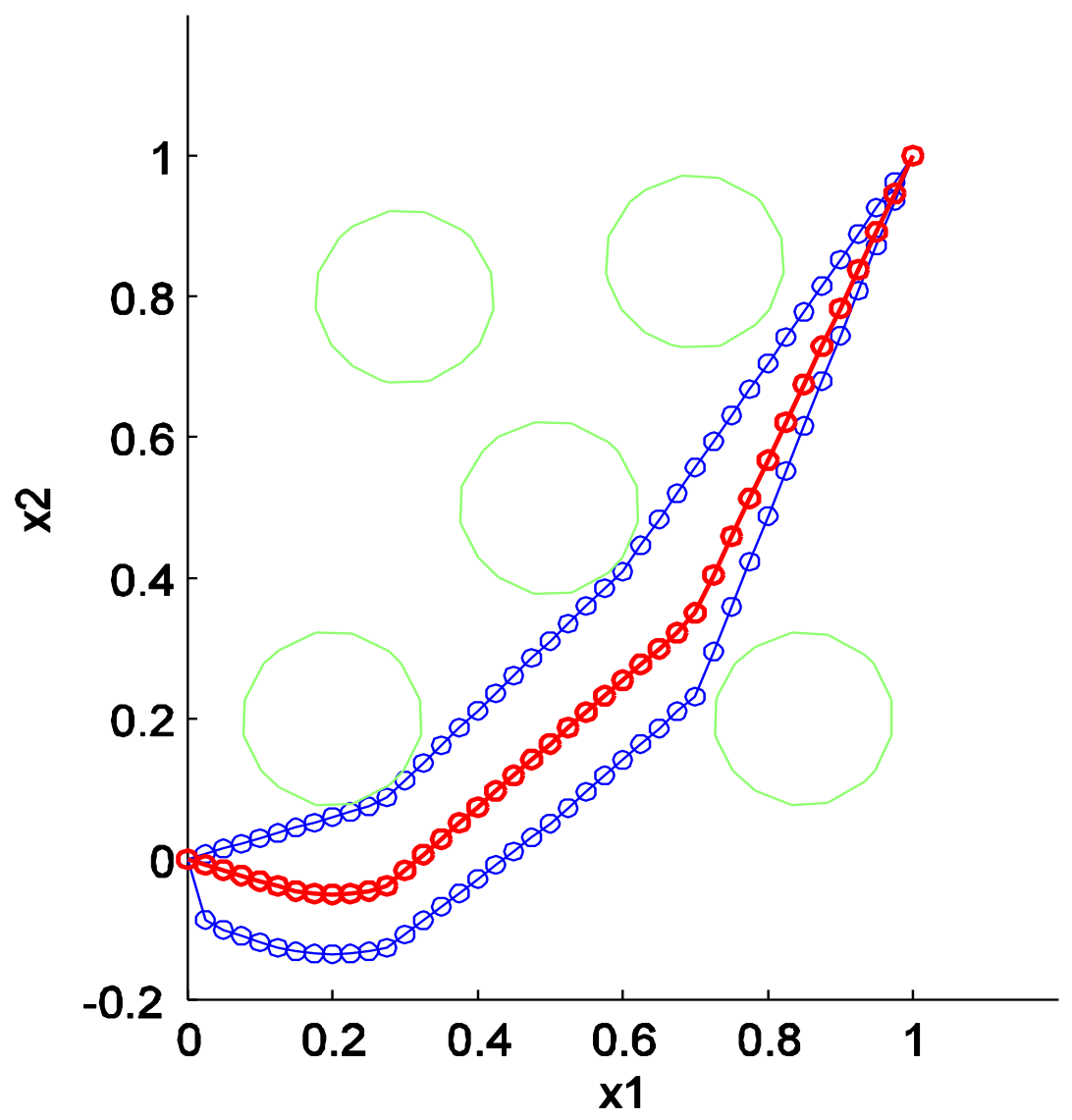




\section{References}

1. J. C. Latombe, Robot motion planning, Kluwer Academic Publishers, Boston, 1991.

2. Y. Wang, D. M. Lane, G. J. Falconer, Two novel approaches for unmannd under water vehicle path planning: constrained optimization and semi-infinite constrained optimization, Robotia 18(2000), 123-142.

3. A. H. Borzabadi, A. V. Kamyad, M. H. Farahi,H. H. Mehne, Solving some optimal path planning based on measure theory, Applied Mathematics and Computation 170(2005) 1418-1435.

4. K. Mittinen, Nonlinear multi-objective optimization, Kluwer Academic, New York,1993.

5. J. L. Kohon, Multi-objective programming and planning, Academic Press: New York, 1985.

6. M. Sakawa, Fuzzy set and interactive multi-objective optimization, Plenum Press: New York, 1993.

7. M. Delgado, J.L. Verdegay, M. A. Vila, A possibility approach for multi-objective programming problems, Kluwer Academic: New York, 1991.

8. J.E. Rubio, Control and optimization, the linear treatment of nonlinear problems, Manchester University Press, Manchester, UK,1986. 